This is the final peer-reviewed accepted manuscript of:

D. Masotti, A. Costanzo, M. Del Prete and V. Rizzoli

"Time-Modulation of Linear Arrays for Real-Time Reconfigurable Wireless Power Transmission"

in IEEE Transactions on Microwave Theory and Techniques, vol. 64, no. 2, pp. 331342

The final published version is available online at:

https://doi.org/10.1109/TMTT.2015.2512275

Rights / License:

The terms and conditions for the reuse of this version of the manuscript are specified in the publishing policy. For all terms of use and more information see the publisher's website.

This item was downloaded from IRIS Università di Bologna (https://cris.unibo.it/)

When citing, please refer to the published version. 


\title{
Time-modulation of Linear Arrays for Real-time Reconfigurable Wireless Power Transmission
}

\author{
Diego Masotti, Member, IEEE, Alessandra Costanzo, Senior Member, IEEE, \\ Massimo Del Prete, Student, IEEE, and Vittorio Rizzoli, Fellow, IEEE
}

\begin{abstract}
This paper proposes a smart wireless power transmission method, based on a two-step procedure, exploiting real-time beaming of time-modulated arrays. The sideband radiation phenomenon, which is usually a drawback of these radiating systems, is favorably used for intentional wireless power transfer (WPT): in a first step, to precise localize the tag to be powered, in the second one, to perform directive WPT. The approach is first theoretically discussed, then the numerical procedure, which integrates full-wave analysis of the antenna array with nonlinear simulation of the modulated nonlinear feeding network, is used to validate the principle of operation and to include nonlinearities and electromagnetic couplings affecting the whole system performance. The procedure allows a flexible design of the TMA-based WPT system, taking into account the impact of different array elements layout and spacing on localization and power transmission performance. Experiment of the first step is carried out in a real indoor environment at $\mathbf{2 . 4 5}$ GHz: a TI MSP430 drives a Schottky-diode-based network to provide proper modulated RF excitations of the array elements. Measurements show that the system is able to select tags to be

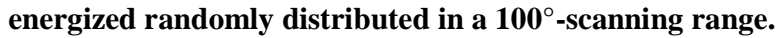

Index Terms-Wireless power transfer, linear antenna arrays, time modulation, nonlinear circuits, electromagnetic analysis.

\section{INTRODUCTION}

$\mathrm{I}^{\mathrm{N}}$ $\mathrm{N}$ modern society there is plenty of applications based on networked low-cost nodes (tags) that are distributed in the space: wireless sensor networks, logistic, industrial automation. The need for low power, battery-less, or rechargeable devices is thus increasing exponentially. The possibility to scavenge the radio-frequency (RF) from the ambient has demonstrated to be a potential solution to the energy autonomy problem of such devices: but the extremely low levels of available RF power in practical scenarios [1] lead to few $\mu \mathrm{W}$ of collected dc power [2], [3]. For this reason wireless power transfer (WPT) is foreseen as one of the key enabling technologies for an energy-aware world [4], for the effective implementation of the so popular paradigm of the

Manuscript received July 21, 2015.

This paper is an extended paper from the IEEE Wireless Power Transfer Conference, Boulder, CO, USA, May 13-15, 2015

D. Masotti and V. Rizzoli are with DEI, University of Bologna, Italy (email:diego.masotti@unibo.it; vittorio.rizzoli@unibo.it).

A. Costanzo and M. Del Prete are with DEI, University of Bologna, Cesena Campus, Italy (e-mail: alessandra.costanzo@unibo.it, massimo. delprete3@unibo.it)
Internet of Things [5]-[7]. With these scenarios in mind, smart strategies to intentionally provide RF energy to a large number of wireless nodes become of leading importance [8]-[11].

This paper proposes a new solution based on a suitable configuration of time-modulated arrays (TMAs), firstly introduced in [12], for wirelessly energizing randomly distributed selectable devices.

The unrivaled level of re-configurability of TMA has been analytically computed by using the time as an additional degree of freedom in radiation pattern synthesis [13], [14]: ideal switches connected to the array ideal elements are periodically modulated to realize real time beaming. Several optimized modulation sequences have been already proposed. Among the most appealing ones, we cite those providing: adaptive nulling [15], harmonic beam-forming [16], [17], beam steering [18], [19], and direction finding [20]-[22]. A dynamic radiating system based on the effective implementation of TMA theory is thus foreseen to be a very promising solution for the above mentioned widely distributed modern wireless applications, such as secure communication in future cognitive radio systems [23].

It is noteworthy that, despite of their agility, TMAs own a much simpler architecture if compared to other beam forming solutions, such as phased-arrays or retrodirective arrays [24], [25]: indeed, the desired phase condition at the antennas ports is reached with no need for phase shifters, thus drastically simplifying the design and the realization phases, as schematically reported in the block diagram of Fig. 1 for a linear TMA.

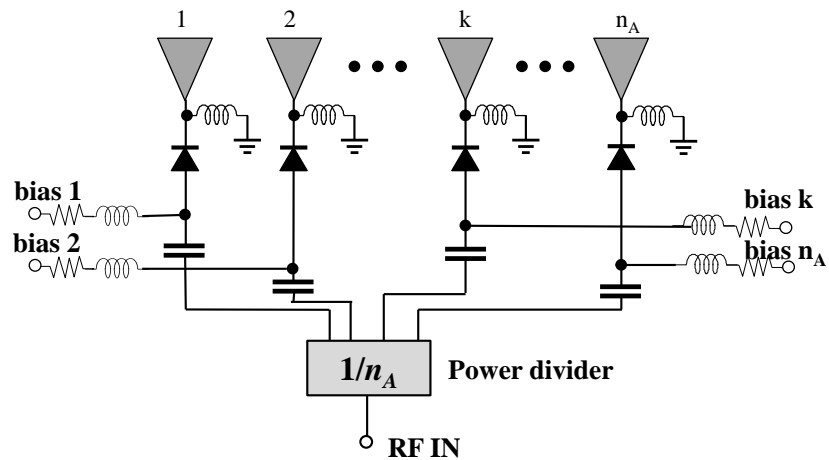

Fig. 1. Schematic representation of a linear, $\mathrm{n}_{\mathrm{A}}$-element TMA, with detailed diodes switch bias networks, including DC-block capacitors.

Few interesting implementations of TMA theory are provided in [26], [27]. In [28], the direction finding operation is demonstrated, in the broadside direction only, by using 
bulky wire monopoles and by adding a coaxial line stretcher at one antenna port for the phase compensation. In [22] the same operation is obtained with planar patches, but a quite complex signal processing is needed.

In this paper we propose a novel two-step real-time beaming implementation of linear TMAs to realize a smart WPT system: in the first step, the TMA is configured in such a way that the sole inner couple of antennas are driven and the control sequences are designed to simultaneously provide the $\Sigma$ and $\Delta$ patterns [29], that allow to precisely determine the position of the devices to be energized; such positions are used in the second operative step of the system, to set the time control modulating signals of the entire array for real-time beaming the RF power to the wanted directions. The dynamic nature of TMAs thus allows creating an agile energy-aware reader/transmitter to be adopted in different scenarios, pervasively occupied by battery-less devices.

Starting from the theoretical motivations introduced in [12], in this paper we first demonstrate the numerical implementation of the entire system design for the two-step operation. This is carried out by means of the multi-domain CAD approach described in [30], which allows to account for the fundamental effects of both nonlinearities and electromagnetic (EM) couplings. Differently from other researches on TMAs, in our approach we consider both real switches and real radiators, driven by various switch control patterns available in the literature. Interesting theoretical optimization strategies have been proposed in [14], [26], [31][34], but under ideal conditions, both from the control and radiation point of view, whereas in [35] a more realistic pulse shape is taken into account during the design procedure. In our case the nonlinear circuit effects, as well as the linear EM ones, are all taken into account in this way, and reveal their importance in the effective implementation of the TMA-based WPT system.

The remainder of the paper is organized as follows. In Section II the main features of TMAs are presented, focusing on favorably exploitation of the sideband radiation phenomenon, which is usually a drawback of these radiating systems, for intentional WPT. Section III briefly recalls the guidelines of our CAD approach, useful for a better understanding of the complex TMA radiation mechanism. Section IV describes the two-step agile WPT strategy, whereas the corresponding simulated results are given in Section $\mathrm{V}$ for two real array layouts. Section VI discusses the experimental results for the first-step operation of the proposed TMA system, in a real indoor environment. A planar $2.45 \mathrm{GHz}$ twomonopole array is realized and a TI MSP430 is used to drive the control pattern of the array Schottky-diode-based feeding network.

\section{TMA RADIATION AT FUNDAMENTAL AND SIDEBAND FREQUENCIES}

The presence, at TMA element ports, of nonlinear switches, driven by periodical control sequences, open the way to an almost unlimited number of excitation combinations: not only the switch on percentage [14], but also the rise and fall instants [26], [31], [32] can be adopted in an optimization procedure to manipulate the high versatility of these radiating system. Fig. 1 shows the simple array architecture for a standard linear array: as can be evinced from the figure, the array architecture is extremely simple, especially if compared to phased-arrays. No complex phase-shifters are requested, but only simple control circuit boards. Moreover, TMAs offer an additional known capability, not feasible for other radiating architectures, represented by a multi-frequency radiation mechanism; and this will represent the focus of the present paper.

Let us start considering a linear array, whose $n_{A}$ elements are resonant at the fundamental frequency $f_{0}$, and are aligned along the direction $\hat{\boldsymbol{a}}(\hat{\boldsymbol{a}} \cdot \hat{\boldsymbol{r}}=\cos \psi)$, with inter-element spacing $\mathrm{L}$. The RF switch at the $\mathrm{i}$-th antenna port, driven by a periodical sequence of rectangular pulses of period $T_{M}=1 / f_{M}$ and normalized amplitude $U_{i}(t)$, leads to the replacement of the generic standard constant excitation coefficient $A_{i}$ with the corresponding time-dependent version $A_{i} \cdot U_{i}(t)$. This way the far-field radiated by the whole array becomes time-dependent, too, through the time-dependency of the array factor $A F$, as described in the following field evaluation in the point $(r, \theta, \phi)$ :

$$
\begin{aligned}
\boldsymbol{E}(r, \theta, \phi, t) & =\boldsymbol{E}_{0}(r, \theta, \phi) \sum_{i=0}^{n_{A}-1} A_{i} U_{i}(t) e^{j i \beta L \cos \psi}= \\
& =\boldsymbol{E}_{\boldsymbol{0}}(r, \theta, \phi) A F(\theta, \phi, t)
\end{aligned}
$$

where $\beta$ is the free space phase constant, and $\boldsymbol{E}_{\boldsymbol{0}}$ represents the far-field radiated at the carrier frequency $f_{0}$ by the baseelement of the array. Due to the periodicity of the switch control sequences it is possible to Fourier-transform the timedependent array factor:

$$
\begin{aligned}
A F(\theta, \phi, t) & =\sum_{h=-\infty}^{\infty} A F_{h}(\theta, \phi, t)= \\
= & \sum_{h=-\infty}^{\infty} e^{j 2 \pi\left(f_{0}+h f_{M}\right) t} \sum_{i=0}^{n_{A}-1} A_{i} u_{h i} e^{j i \beta L \cos \psi}
\end{aligned}
$$

where $u_{h i}$ is the h-th Fourier coefficient of the $U_{i}(t)$ pulse.

The direct consequence of the replacement of (2) in (1) is the multi-frequency radiation contribution: indeed, the TMA is able to radiate at the usual fundamental frequency $f_{0}(h=0)$, but also at the near-carrier sideband harmonics $f_{0} \pm h f_{M}(h= \pm 1$, $\pm 2, \ldots)$. This sideband radiation is efficiently transmitted/ received due to the low value of the modulation frequency $f_{M}$ with respect to the carrier $f_{0}(\mathrm{MHz}$ vs. $\mathrm{GHz})$, which allows the array elements to be still almost resonant.

At the beginning of TMAs history, the phenomenon of sideband radiation has represented an unwanted radiation. Many efforts have been made in literature to suppress this waste of power: Fig. 2 gives, at a glance, the effect of this phenomenon by plotting the radiation patterns of a 16 -element linear array operating at $2.45 \mathrm{GHz}$, driven by a control sequence assuring both a sideband radiation level (SBL) around $-20 \mathrm{~dB}$, and a side-lobe-level (SLL) at the same frequency below $-30 \mathrm{~dB}[31]$. 
In recent years, the capability of TMAs to simultaneously radiate at different frequencies has been considered as an added value for specific applications. In this paper we intend to exploit the capabilities of direction finding [20]-[22] and harmonic-beamforming [16], [17] to realize a new and smart WPT procedure, as explained in Section IV.

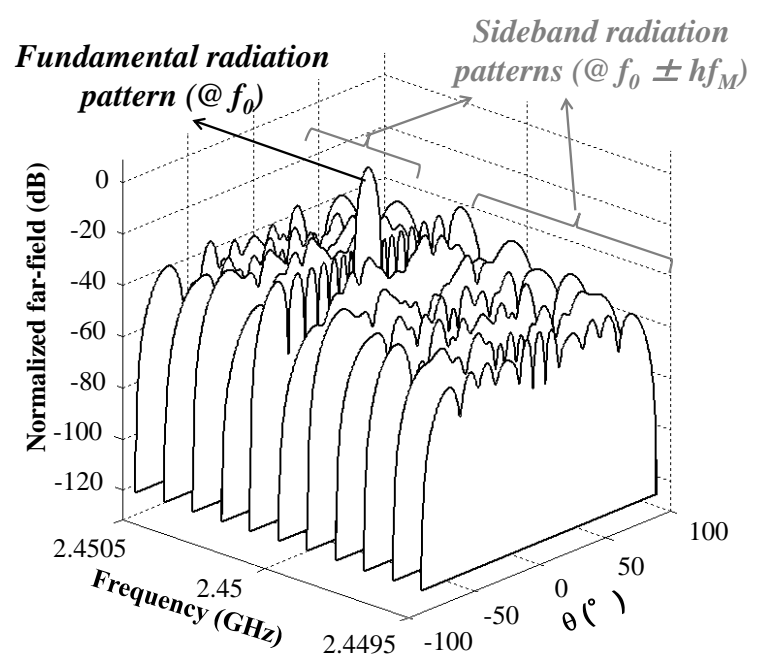

Fig. 2. Multi-frequency radiation patterns of a 16-element linear TMA, with switch control sequence taken from [31].

\section{NONLINEAR AND FULl-WAVE Co-SimUlation OF TMAS}

The complexity of TMA systems, with the unavoidable presence of EM couplings and the nonlinear nature of the driving switches, can lead to highly inaccurate results if proper countermeasures are not taken. The analytical approaches available in literature focus on the fundamental goal of the control sequences optimization, but consider both ideal radiators and ideal switches. For this reason we have proposed in [30] a rigorous approach for the accurate modelling of TMA systems, by accounting for all the non-idealities. In this paper we adopt the same simulation tool, and some hints are restated here, for the sake of completeness and for a better understanding of the following results.

The approach consists of the nonlinear/EM analysis of the whole radiating system by combining the Harmonic Balance (HB) technique, for the accurate description of the nonlinearities, and the broadband full-wave simulation of the array and its feeding network. In case of an array with $n_{A}$ ports (identical to the number of switches) the EM-based description of the system will consists of a $n_{A}+1$-port network (including the RF input port) where the $n_{A}$ switch ports are internal ports of the EM simulator, i.e., couples of floating nodes directly connected to the mesh, thus embedding the diodes in the whole EM-based system.

As described by Fig. 3, where three different switch controls are given, the superposition of the periodic switch modulation to the carrier frequency $f_{0}$ to be radiated, allows to resort to the modulation-oriented HB method [36]: since the condition $f_{0} \gg f_{M}$ is always valid in practical TMA applications ( $\mathrm{GHz}$ vs. a few $\mathrm{MHz}$ ), the modulated regime can be seen as a sequence of slowly-changing unmodulated ones.

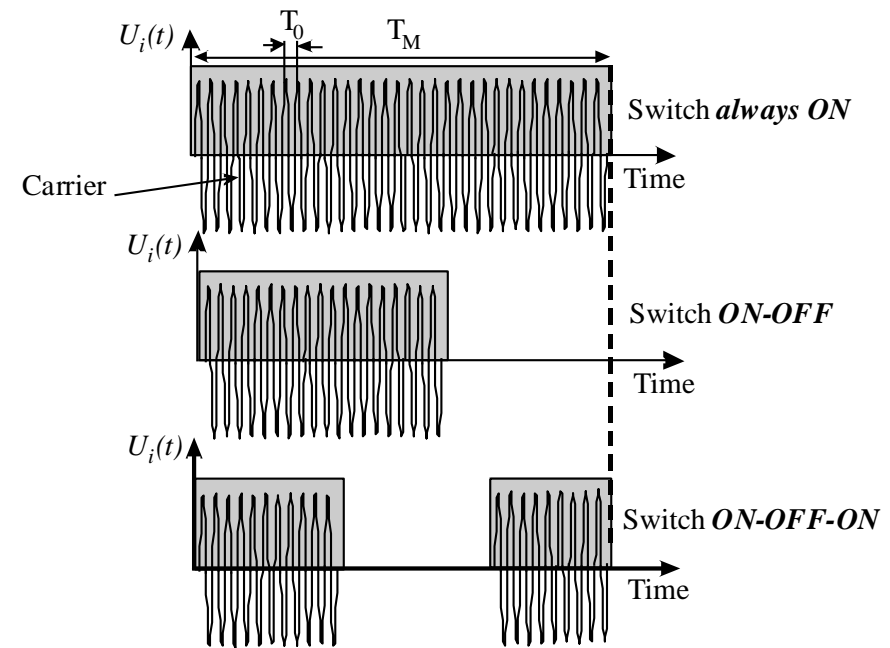

Fig. 3. Examples of periodical switches excitation sequences, modulating the RF carrier waveforms, for three different switching patterns.

Therefore a generic signal flowing in the circuit (e.g. the current at the i-th port of the array) can be cast in the form:

$\mathrm{i}_{\mathrm{A}}{ }^{(\mathrm{i})}\left(t, t_{M}\right)=\operatorname{Re}\left[\sum_{k=0}^{n_{H}} \boldsymbol{I}_{A, k}^{(i)}\left(t_{M}\right) \exp \left(j k 2 \pi f_{0} t\right)\right]$

where $n_{H}$ is the needed number of harmonics of the fundamental frequency $f_{0}, t_{M}$ is the slow envelope time of the switches modulation law, $t$ is the fast carrier time, and $\boldsymbol{I}_{A, k}^{(i)}\left(t_{M}\right)$ is the time-dependent complex $k$-th current envelope (or modulation law). For the far-field evaluation two aspects have to be considered: i) the modulation is obviously transferred from the driving currents to the radiated far-field; ii) the linearity of the antenna system allows to exploit the superposition principle and to calculate the field from the knowledge of the excitations (currents): thus the far-field envelope at the generic harmonic $k$ takes on the following expression [37]:

$$
\begin{aligned}
& \mathbf{E}_{k}\left(r, \theta, \phi ; t_{M}\right)=\frac{\exp (-j \beta r)}{r} \bullet \\
& \bullet \sum_{i=1}^{n_{A}}\left[\hat{\boldsymbol{\theta}} B_{\theta}^{(i)}\left(\theta, \phi ; k \omega_{0}\right)+\hat{\boldsymbol{\phi}} B_{\phi}^{(i)}\left(\theta, \phi ; k \omega_{0}\right)\right] I_{A, k}^{(i)}\left(t_{M}\right)- \\
& -j \frac{1}{r}\left[\left.\sum_{i=1}^{n_{A}} \frac{\partial\left\{\exp (-j \beta r)\left[\hat{\theta} B_{\theta}^{(i)}\left(\theta, \phi ; k \omega_{0}\right)+\hat{\phi} B_{\phi}^{(i)}\left(\theta, \phi ; k \omega_{0}\right)\right]\right\}}{\partial \omega}\right|_{\omega=k \omega_{0}} \bullet\right. \\
& \left.\bullet \frac{d I_{A, k}^{(i)}\left(t_{M}\right)}{d t_{M}}\right]
\end{aligned}
$$

where $B_{\theta}^{(i)}, B_{\phi}^{(i)}$ are the scalar components of the normalized field, easily evaluated by means of a sequence of full-wave analyses: for each array port, the generic $i$-th radiating element $\left(i=1, \ldots, n_{A}\right)$ is excited by a unit-current sinusoidal source of frequency $k f_{0}$, while the other ports are left open. The additional term in square brackets is due to the timedependency of the current phasors. It is worth mentioning that, given for granted the antenna topology, this time-consuming EM database has to be evaluated only once in the frequency bands of interest; it can then be used whatever antenna regime 
need be computed.

The set of results, in terms of radiated far-field, given by the present approach, is a complete one, since the field is treated as any other signal in the system.

The direct application of (4) provides the field envelope at the desired harmonic (typically the fundamental, $k=1$ ), in any direction of radiation. Hence, from (4), the far-field harmonics of the bi-dimensional regime due to the intermodulation between $f_{0}$ and $f_{M}$ is available: $\boldsymbol{E}_{k h}(r, \theta, \phi)$. While, by varying the harmonic number $h$, the resulting far-field spectrum around the carrier harmonic $k$ is obtained, for a fixed distance/direction. Finally, by varying $(\theta, \phi)$, the result is the radiation surface/pattern at a given distance $r$ and for a given harmonic.

An important aspect of this approach is that it allows to investigate the nonlinear nature of the radiating system. In the following the power transmission as a function of the input power level will be discussed in detail, by putting into evidence the power range where the desired radiating performance can be achieved.

\section{TWO-STEP AgILE WPT STRATEGY}

The scenario we have in mind is an indoor room where several sensors, equipped with rectennas, are randomly placed. The dynamic re-configurability of a linear TMA is fully deployed in a novel WPT two-step procedure, as represented in Fig. 4: in the first step only two elements of the TMA are periodically driven to localize the $N_{\text {tag }}$ tags; in the second step the entire $n_{A}$-element array is used to precisely energize the previously detected tags. In both phases we take advantage from the TMA capability to perform a multiple radiation at the fundamental $f_{0}$ and the first sideband harmonics $f_{0} \pm f_{M}$.

\section{A. Localization Step}

By properly driving the two switches, an interesting direction finding functionality of a two-element TMA is deployable exploiting the TMA sideband radiation. We choose a complementary control sequence of the kind reported in Fig. 5 with solid lines (i.e. duty cycle of $50 \%$ for both switches) to obtain from the two-element array symmetric radiation surfaces both of the sum-type $(\Sigma)$ at $f_{0}$ (two elements in phase), and of the difference-type $(\Delta)$, but at $f_{0} \pm f_{M}$ (two elements out of phase). Fig. 5 also shows the tuning parameter $d$ allowing a variable overlapping percentage of the control sequences: in

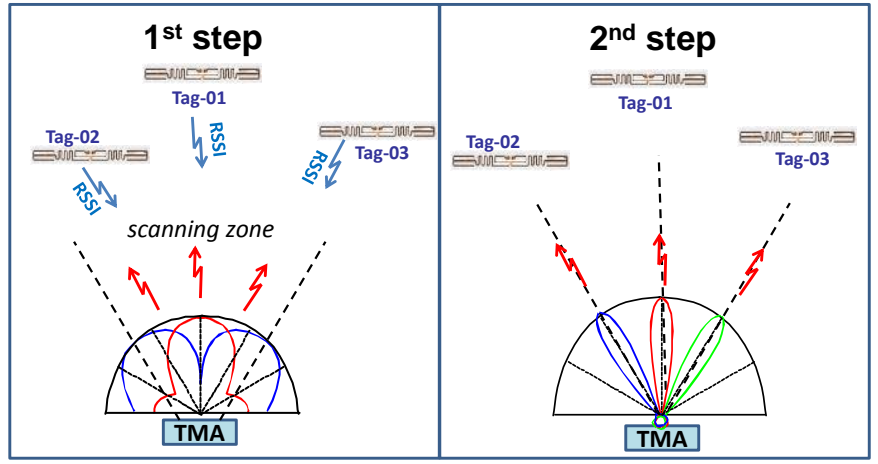

Fig. 4. Two-step WPT procedure exploiting a linear TMA. $1^{\text {st }}$ step: tags localization; $2^{\text {nd }}$ step: power transmission to the previously detected tags. this way beam steering of the $\Delta$ pattern is achieved in the scanning plane $\left(-90^{\circ} \leq \theta \leq 90\right)$ : increasing $\mathrm{d}$ results in a shift increase of the $\Delta$ null in the right half-plane $\left(0^{\circ} \leq \theta \leq 90\right)$ at $f_{0}$ - $f_{M}$; a symmetric result in the left half-plane $\left(-90^{\circ} \leq \theta \leq 0\right)$ is observed at $f_{0}+f_{M}$.

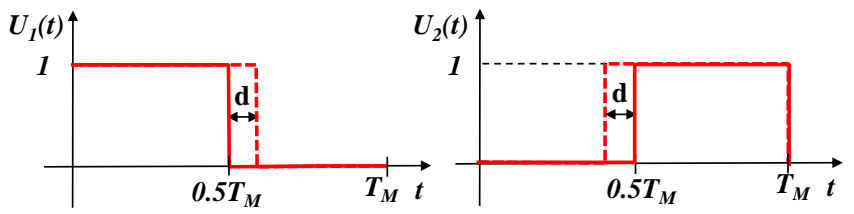

Fig. 5. Switches driving sequences of a two-element array for localization purposes: $\mathrm{d}$ is the pulse shape design parameter, for $\Delta$ pattern steering.

Steering $\Delta$-pattern is made possible by the complex nature of the corresponding Fourier coefficient $u_{ \pm I i}$ in (2). Conversely, the real coefficient $u_{0 i}$ is responsible of the fixed nature of the $\Sigma$-pattern, while changing d. Fig. 6(a) shows the radiation patterns in the scanning plane of an ideal array of two isotropic radiators, with $\mathrm{L}=\lambda / 2$ : they thus correspond to the $A F$ patterns obtained from (2) with $U_{i}(t)(i=1,2)$ given by the waveforms of Fig. 5. With an increase of $d$ up to $20 \%$, a scanning region of about $\pm 60^{\circ}$ is achieved.

In [20] a reduced element spacing $L$ is suggested: in this way the goal of increasing the $\Sigma$-pattern flatness is obtained, and consequently almost unchanged localization properties are guaranteed, while steering. In Fig. 6(b) the same ideal patterns as in Fig. 6(a) are reported, with $\mathrm{L}=\lambda / 8$. Superior scanning performance pertain to this case: besides the almost flat $\Sigma$ pattern, a wider scanning region of about $\pm 70^{\circ}$ with sensibly lower values of $d$ is possible.

Let us assume that sensors IDs have been already acquired by standard RFID reading operations of passive tags. The (flat) $\Sigma$ pattern of a standard (un-modulated) two-element array could be used for this purpose. When tag IDs are

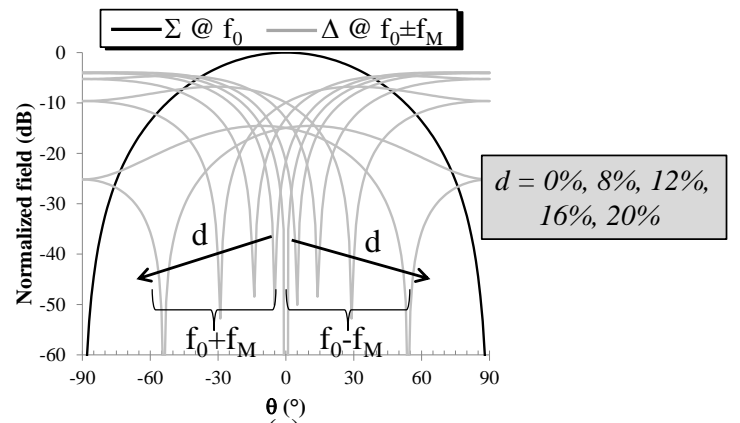

(a)

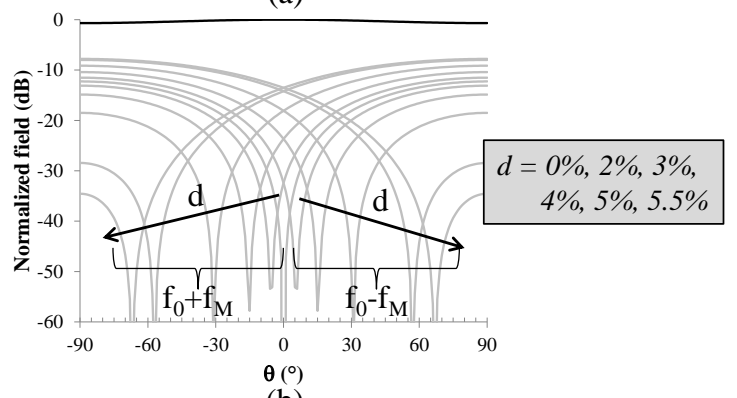

(b)

Fig. 6. Fixed $\Sigma$ and steerable $\Delta$ patterns of an ideal array of two isotropic elements (a) with spacing $\lambda / 2$, and (b) with spacing $\lambda / 8$, as a function of $d$. 
acquired the true scanning phase takes place, by exploiting the time-modulation according to the patterns of Fig. 5: the sharp nulls of the steered $\Delta$ patterns allow high resolution in the tags detection: the backscattered Received Signal Strength Indicators (RSSI) due to the $\Sigma$ and $\Delta$ patterns can be suitably combined to build the Maximum Power Ratio (MPR) [29]:

$$
\operatorname{MPR}\left(\theta_{j}\right)=\Sigma_{R S S I}^{d B}\left(\theta_{j}\right)-\Delta_{R S S I}^{d B}\left(\theta_{j}\right) \quad ; \quad\left(j=1,2, \ldots, N_{t a g}\right)
$$

The combination of the radar monopulse operating principle with the scanning capability has proven its effectiveness in indoor localization, with resolution up to few $\mathrm{cm}$ at $2.45 \mathrm{GHz}$ [29]. At the end of the scanning activity a vector with the $N_{\text {tag }}$ values of $\theta$ corresponding to the peaks of the received MPRs $\left(\theta_{\text {peak }}\right)$ is recorded.

\section{B. Power Transfer Step}

Once the tags positions have been detected, the entire array is driven by a proper control sequence involving all the switches and providing the desired radiation properties: from this side, there is plenty of optimization strategies for the engineering of the radiation patterns shape of ideal TMAs [26], [31]-[34]. In particular, it is possible to add, as additional design parameter, the maximum radiation direction at the sideband harmonics $f_{0} \pm h f_{M}(h \neq 0)$. In this way it is possible to point the sideband harmonic radiation in the desired direction, and this can be done simultaneously at several near-carrier frequencies $(h \geq 1)$.

A possible decision rule during the WPT activity could be to split the scanning region (the actual situation implementing Fig. 6) into sectors of amplitude equal to the half power beam width (HPBW), centered around $\theta_{H P B W}$, as described in Fig. 7: here, the hypothetical situation of an array of 16 dipoles, with $\mathrm{HPBW}=7^{\circ}$, and the exploitation of up to the sixth sideband harmonics, is envisaged. For a $\theta_{\text {peak }}$ falling in the $\theta_{\mathrm{HPBW}^{-}}$ centered sector, the pre-loaded control sequence pointing the desired harmonic pattern (at $f_{0} \pm h f_{M}$ ) to the $\theta_{H P B W}$ direction is used. This operation is repeated for each $\theta_{\text {peak }}$. In case of a $\theta_{\text {peak }}$ falling in the sector centered around $\theta_{H P B W}=0^{\circ}$, the fixed fundamental harmonic beam can be used to energize the corresponding sensor, while the first sideband harmonics can simultaneously transfer the power to another couple of almost

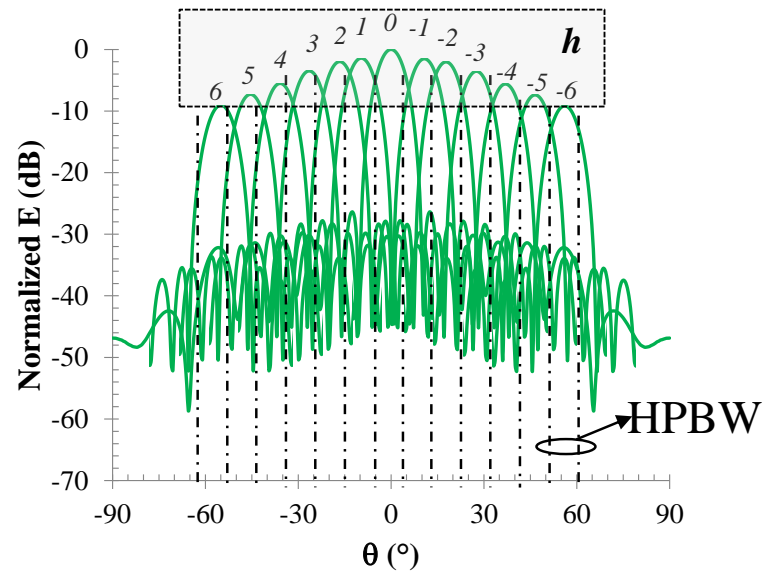

Fig. 7. Ideal full exploitation of a 16-dipole TMA sideband radiation, when considering up to the sixth harmonic, for multiple tags powering. symmetrically placed tags.

\section{Simulation Results}

The CAD approach described in Section III can now help in the choice of the proper antenna topology from both the localization and power transmission points of view, by evaluating plots of the kind of Figs. 6 and 7, now obtained by taking into account the actual layouts and radiation patterns.

\section{A. Localization Step}

For localization purposes we examine the planar, twoelement array topologies described in Fig. 8: a couple of monopoles with $\mathrm{L}=\lambda / 2$ and $\mathrm{L}=\lambda / 8$, and a couple of multilayer, square patch antennas, fed by means of apertures in the ground plane, with $L=\lambda / 2$ and $L=\lambda / 3$. All the arrays are realized on a Taconic RF60A substrate $\left(\varepsilon_{\mathrm{r}}=6.15\right.$, thickness $=0.635 \mathrm{~mm}$ ), and the corresponding antennas resonate at $f_{0}=2.45 \mathrm{GHz}$. Note that in order to experimentally validate, in a research lab, the numerical results discussed in this section we have chosen to use medium-power microwave Schottky diodes (Skyworks SMS7630-079), as the switching elements, driven by periodic sequences with modulation frequency $f_{M}=25 \mathrm{kHz}$.

The linear localization performance of the arrays under test, are then analyzed at low power levels: a $-10 \mathrm{dBm}$ RF power at each antenna port is used for the following analyses. It is noteworthy that, for higher power needs, PIN diodes (such as Infineon BAR64-02V) and their associated nonlinear models can be adopted in a straightforward way to predict the system behavior.

Note that the arrays of Fig. 8 correspond to the inner elements of larger arrays, deploying a higher number of radiating elements, needed for the second step of the WPT procedure. The following results are thus obtained by making use of the EM-database $\left(B_{\theta}{ }_{\theta}^{(i)}, B_{\phi}{ }^{(i)}\right)$ corresponding to the larger arrays (with $n_{A}$-ports, $n_{A}=16$ ). This way, the unavoidable EM-couplings between the nearby elements are automatically taken into account, even if the sole two inner switches are driven at this stage, while the remaining $n_{A}-2$ ones are left

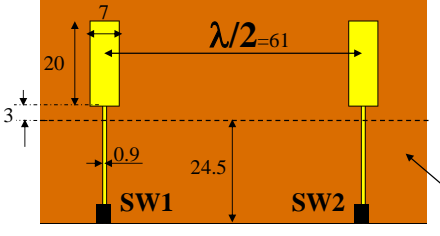

(a)

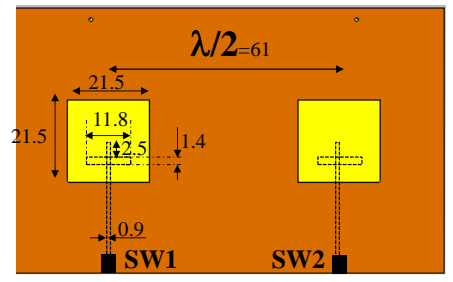

(c)

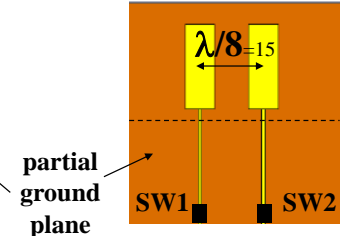

(b)

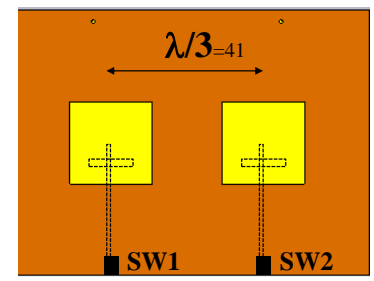

(d)
Fig. 8. Layouts and dimensions (in $\mathrm{mm}$ ) of planar two-element TMAs with: (a) $\lambda / 2$-spaced monopoles, (b) $\lambda / 8$-spaced monopoles, (c) multilayer $\lambda / 2$ spaced patches; (d) multilayer $\lambda / 3$-spaced patches. 
open. Moreover the same database can be exploited in the analyses involving all the $n_{A}$ switches of the second phase.

Fig. 9 shows the results in terms of $\Sigma$ and $\Delta$ radiation patterns for the arrays of Fig. 8 in the scanning $\theta$-plane, for different d-values: very wide steering of the sideband patterns can be easily obtained with the monopoles arrays, as shown in Fig. 9(a, b). A slightly flatter $\Sigma$ pattern is achieved in the closer antennas case, as expected; but the actual arrays radiation patterns taken into account in this analysis, drastically worsen the closer antennas behavior with respect to the ideal case of Fig. 6(b). In both the monopole array cases the scanning region remains almost unchanged: roughly $-50^{\circ} \leq$ $\theta \leq 50^{\circ}$. It is worth noting also the higher $\mathrm{d}$ parameter value needed for these steering activities: $\mathrm{d}=32 \%$ determines $\Delta$ peaks (at $f_{0} \pm f_{M}$ ) steering at $\pm 45^{\circ}$ : besides the non-ideal antenna radiating characteristics, the real control switch behavior influences the final array performance, too. Finally, the results of Fig. 9 (c, d) demonstrate that the more directive patch antenna behavior is a drawback in this case. The scanning region is drastically reduced: more than $50 \%$, with respect to the almost omnidirectional monopoles situation, in the case of closer patches.

A further interesting advantage in using TMAs is represented by the sharpness of the negative peaks of the $\Delta$ patterns, clearly visible in Fig. 9 for all the array topologies under exam. These peaks are improved by the timemodulation technique, because in this way the two antennas are always alternatively active in the $\mathrm{d}=0 \%$ case, or simultaneously active for brief time intervals in the $\mathrm{d}>0 \%$ cases. This configuration strongly reduces the EM couplings between the two antennas, and drastically improve the whole system performance, with respect to an identical standard array. This consideration has been validated by the full-wave simulation: the array of Fig. 8(a) has been described as a two-

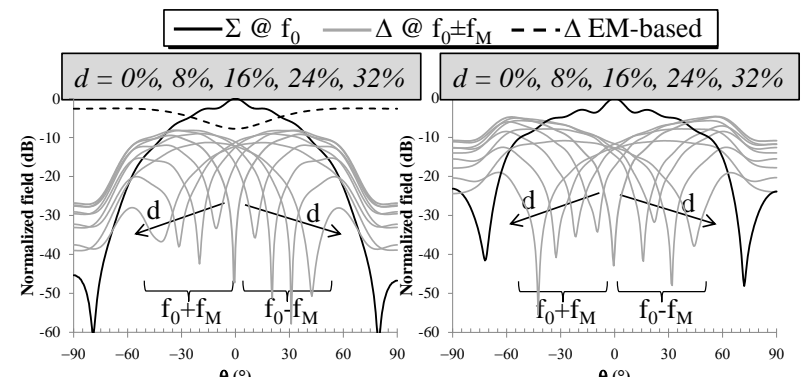

(a)

(b)

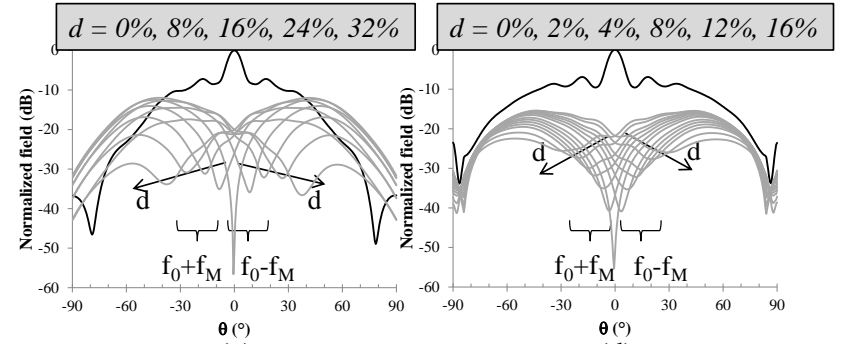

(c)

(d)

Fig. 9. $\Sigma$ and $\Delta$ radiation patterns for the arrays of Fig. 8 in the scanning $\theta$ plane given in the same order, for different d-values. The dotted curve represents the standard $\Delta$ radiation pattern of the array of Fig. 8 (a) with outof-phase antennas excitation. port array, with the ports placed at the diodes location. The $\Delta$ pattern obtained by simply exciting out-of-phase these ports is superimposed to the simulated patterns of Fig. 9(a) by dotted lines: if compared with the $\Delta$-pattern provided by the TMA, in the $\mathrm{d}=0 \%$ case, the full-wave result (dashed black line) has a stronger field, but the sharpness of the peak, which the localization capability relies on, has become significantly worse. Of course, this phenomenon is more evident for omnidirectional antennas which are responsible for high EM couplings: the directive behavior of patch antennas is an advantage, in this case, as verified by full-wave simulation.

\section{B. Power Transfer Step}

In the power transfer step we need a highly directive array in order to precisely energize the detected tags. For this purpose, a standard choice of $L=\lambda / 2$ element-spacing would be recommended: in fact lowering $\mathrm{L}$ implies a reduced-space solution, but also a significant directivity reduction. For this reason, and for the previous results in terms of tags detection, in the following we will limit the comparison between the two 16-monopole arrays of Fig. 10 (a, b), having almost identical directivity of about $14.5 \mathrm{dBi}$ : a standard equally-spaced array, and an unequally-spaced one, with the sole inner couple of monopoles at a reciprocal distance of $\lambda / 8$.

As regards the switches excitation we make use of the sequence proposed in [17] for a 16-ideal-dipole array, reported in Fig. 11, for the sake of clarity.

Fig. 12 shows the obtained harmonics radiation patterns, limited to the $h=0,1,-1$ cases, for the two arrays of Fig. 10: these performance show that this TMA configuration allows to correctly locate three tags, within sectors centered around $\theta_{H P B W}=-30^{\circ}, 0^{\circ}, 30^{\circ}$. Despite the sequence has been

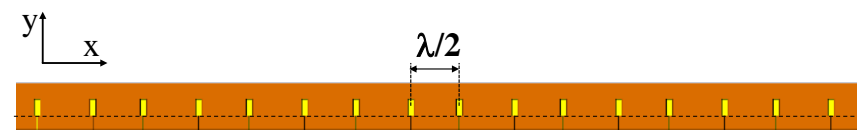

(a)

$\stackrel{\lambda / 2}{\lambda / 2} \rightarrow \stackrel{\lambda / 8}{\longrightarrow}$

(b)

Fig. 10. (a) equally- and (b) unequally-spaced arrays of 16 planar monopoles.

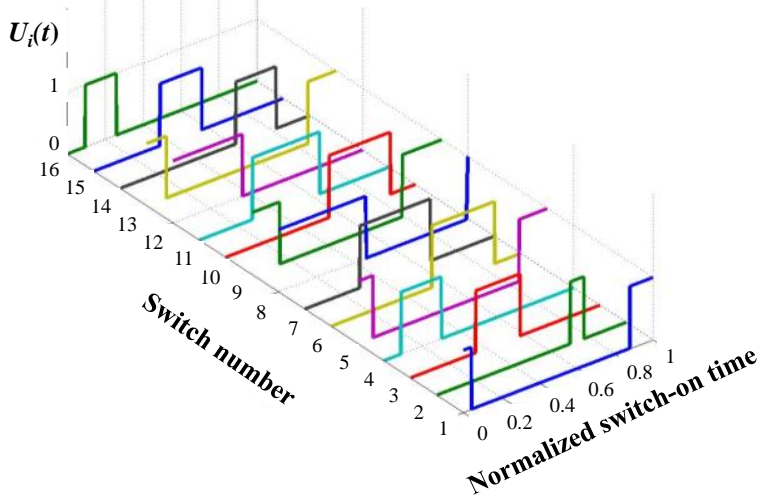

Fig. 11. Switches control pattern for the 16-dipole arrays of Fig. 10, taken from [17]. 
optimized for ideal, uniformly distributed dipoles, the performance are quite satisfactory for the two array arrangements of Fig. 10: with respect to the equally-spaced case (Fig. 12(a)), the unequally-spaced array (Fig. 12(b)) shows a SLL increase of almost $5 \mathrm{~dB}$ for all the plotted patterns: hence the control sequence should be re-optimized taking into account the actual non-standard array layout of Fig. 10(b).
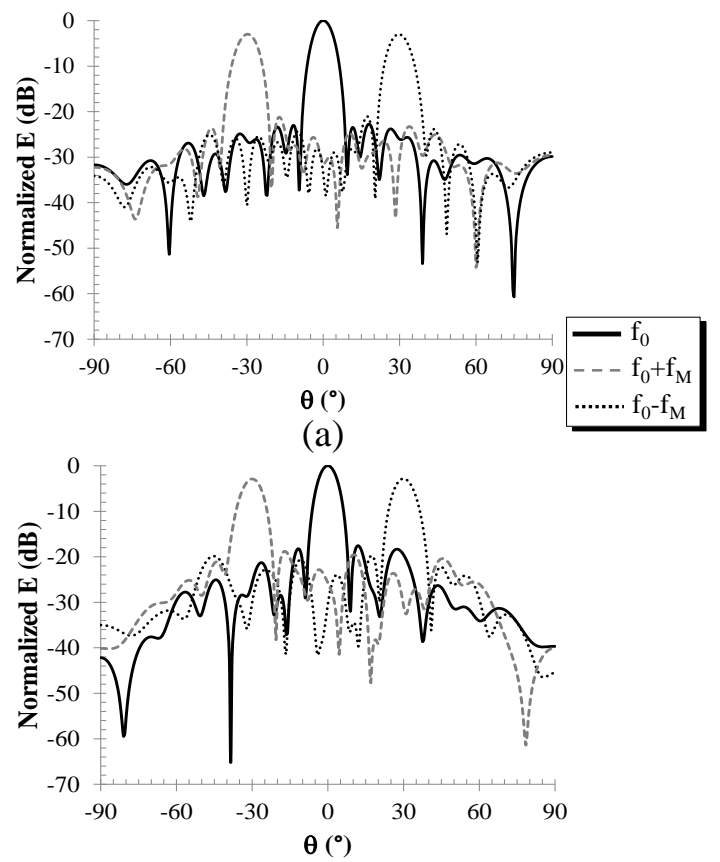

(b)

Fig. 12. Radiation patterns at the fundamental and at the two first symmetrical sideband harmonics, due to the excitation of Fig. 11, (a) for the equally-spaced and (b) for the unequally-spaced arrays of Fig. 10.

By exploiting (4), the far-field envelopes at the fundamental harmonic $(k=1)$ in three directions $\left(\theta=-30^{\circ}, 0^{\circ}, 30^{\circ}\right)$, at a distance $\mathrm{r}=1 \mathrm{~m}$, can be straightforwardly obtained, as reported in Fig. 13 for the array of Fig. 10(a), driven by the sequence of Fig. 11. The waveforms undergo a field intensity oscillation due to the number of active switches at each sampling instant, which is typical of TMAs radiating behavior. These oscillations can be eventually minimized by adopting a proper control sequence, focusing on a flat directivity envelope [38].

In the power transfer step, a transmission of high power levels can occur, if requested by the specific application: e.g. in cases of distant and/or power-hungry tags. For this reason, an investigation of the effects of the input power level on the TMA nonlinear performance can be useful. When the RF input signal increases, the diodes adopted for the switching operations start rectifying a significant portion of the RF signal, in such a way that the bias control sequence can be completely overrun. Once again, this nonlinear phenomenon can be straightforwardly predicted by our CAD procedure, as plotted in Fig. 14, where the equally-spaced array radiation patterns at the three harmonics of interest $(h=0,1,-1)$ are shown for different values of the RF power at each antenna

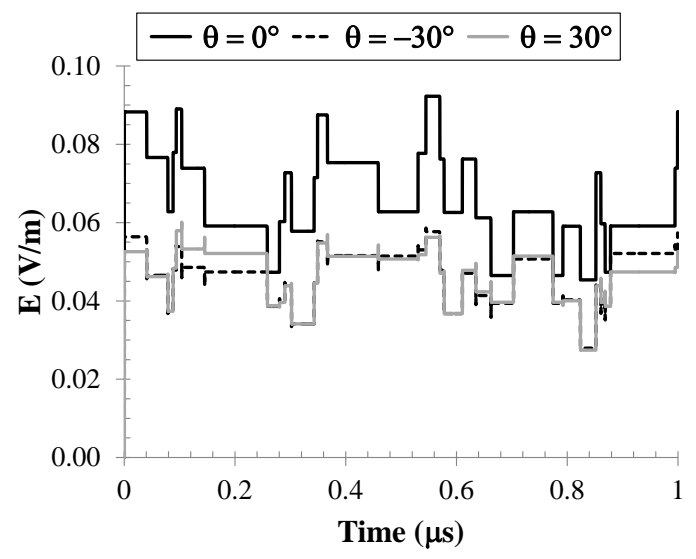

Fig. 13. Far-field envelopes at $1 \mathrm{~m}$-distance, in correspondence of the three radiation maxima of Fig. 12(a).

port. These results are obtained with the time sequence of Fig. 11. The progressive increase of the SLL in the fundamental pattern of Fig. 14(a) with input power $\left(\mathrm{P}_{\mathrm{IN}}\right)$ is evident. For the adopted device this degradation is reached at $\mathrm{P}_{\mathrm{IN}}=10 \mathrm{dBm}$ : all the switches are forced to the on state for the whole modulation period $\mathrm{T}_{\mathrm{M}}$ by this rectification phenomenon. Indeed in such nonlinear condition our nonlinear/EM simulation predicts the same radiation pattern as the one of a standard 16-element static array. The situation is even more
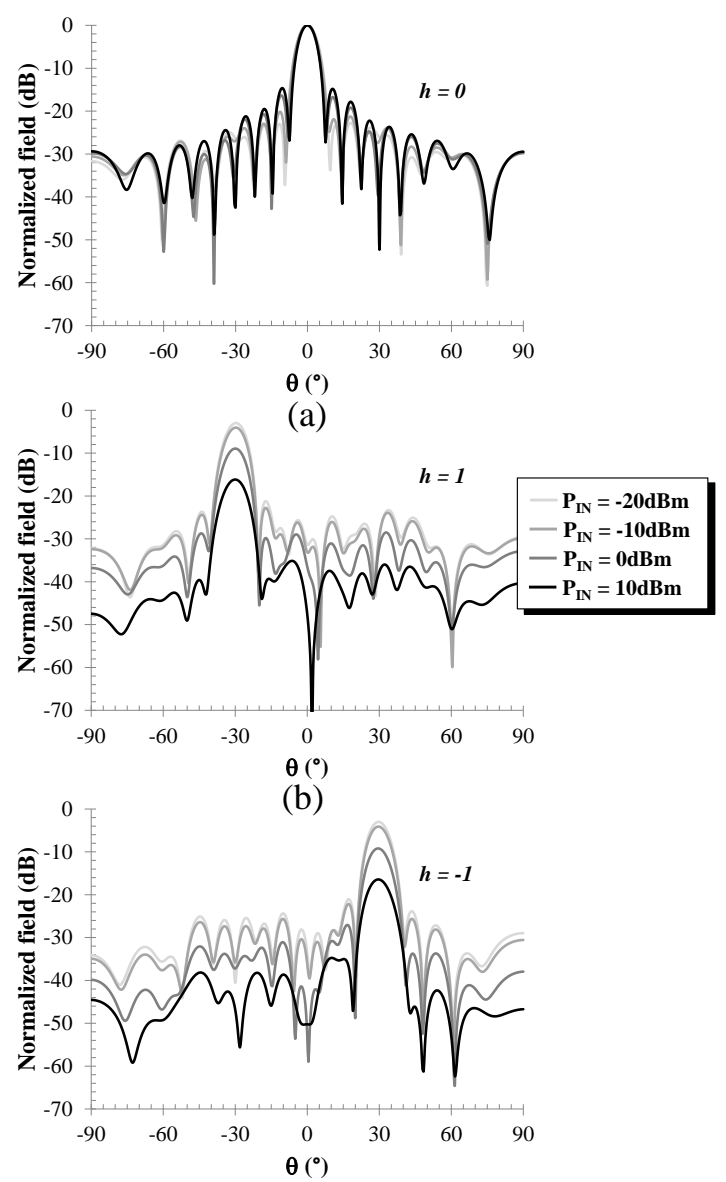

(c)

Fig. 14. Effect of input RF power level on the time-modulated radiation patterns of the array of Fig. 10 (a): (a) at the fundamental and (b, c) at the sideband harmonics. 
shattered for the sideband harmonics patterns $(h= \pm 1)$, where the main lobe radiation strength at $\theta= \pm 30^{\circ}$ is progressively reduced, down to level comparable to the side lobes of the fundamental pattern. This investigation confirms that, for the medium-power Schottky diodes in use, the input power limit is about $0 \mathrm{dBm}$. As previously stated, for higher power needs, the use of different devices can release this limit.

\section{Null mean-value control sequence}

As a further proof of the versatility of these radiating systems, we can also modify the shape of the control pulses in order to limit either the sideband or the fundamental radiations. The sideband radiation suppression becomes advantageous in those scenarios where the tags to be energized fall in the $\theta_{H P B W}=0^{\circ}$ sector, only. Many solutions have been presented in literature to provide a broadside radiation at the fundamental, with simultaneous SBR control [27], [39]. Conversely, for WPT purposes, if no tags are present in the broadside sector $\left(\theta_{H P B W}=0^{\circ}\right)$, in that direction it is required not to waste power. We adopt a very simple solution by designing an anti-symmetric control sequence of the kind reported in Fig. 15(a), where the time intervals with positive and negative values of the bias pulses are identical, as addressed in [12], [40]. In this way, a null mean-value sequence is obtained, thus automatically providing no broadside radiation at $f_{0}$. This can be easily explained by the evaluation of the Fourier coefficient at the fundamental $(h=0)$ in (2):

$U_{i}(t)=\sum_{h=-\infty}^{\infty} u_{h i} \exp \left(j h 2 \pi f_{M} t\right) \Rightarrow u_{0 i}=\frac{1}{T_{M}} \int_{0}^{T_{M}} U_{i}(t) d t=0$

Note that a further degree of freedom is available in this case, given by the positive and negative pulses separation parameter $\tau^{*}$ (Fig. 15(a)), thus increasing the control sequence combinations for future optimizations. The circuit solution requires a more complex implementation, due to a couple of anti-phase switches, as indicated in Fig. 15(b): the diode which is on during the negative cycle requires an additional half wavelength-long meandered line to guarantee the suitable current phase condition at the antenna port, for both positive and negative bias cycles. According to this solution, a symmetric radiation pattern involving only the sideband harmonics $f_{0} \pm f_{M}$ is obtained, and no radiation content is observed at the fundamental harmonic. The optimized suboptimal (with $\tau^{*}=0$ ) anti-symmetric control sequence is shown

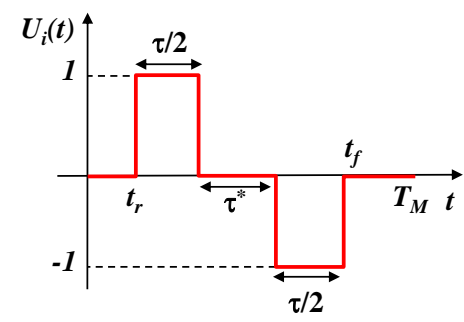

(a)

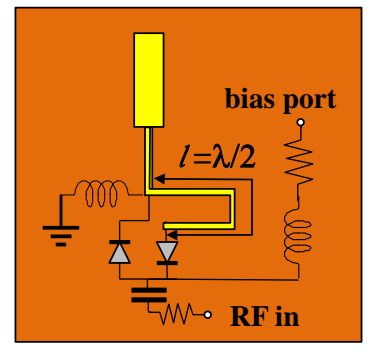

(b)
Fig. 15. (a) Waveform of a null mean-value pulse for broadside radiation suppression at the fundamental frequency; (b) corresponding antenna feeding network schematic layout. in Fig. 16. In case of application of the sequence to the equally-spaced array of Fig. 10(a), the radiation patterns at $f_{0} \pm$ $f_{M}$, shown in Fig. 17, are obtained: two maxima of transmission in correspondence of the sectors $\theta_{H P B W}= \pm 21^{\circ}$ can be exploited in this case, whereas the power radiated at the fundamental remains below the SLL.

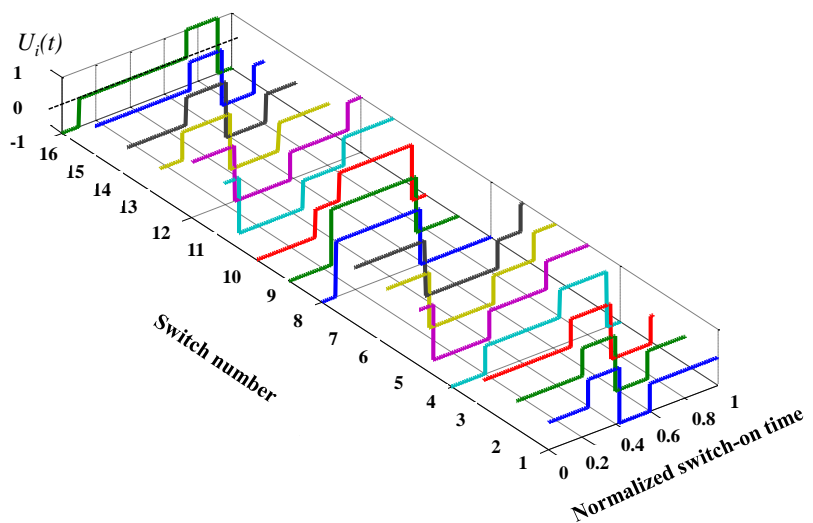

Fig. 16. Switches control pattern realizing null mean value, optimized for WPT purposes, for the 16-dipole array of Fig. 10(a).

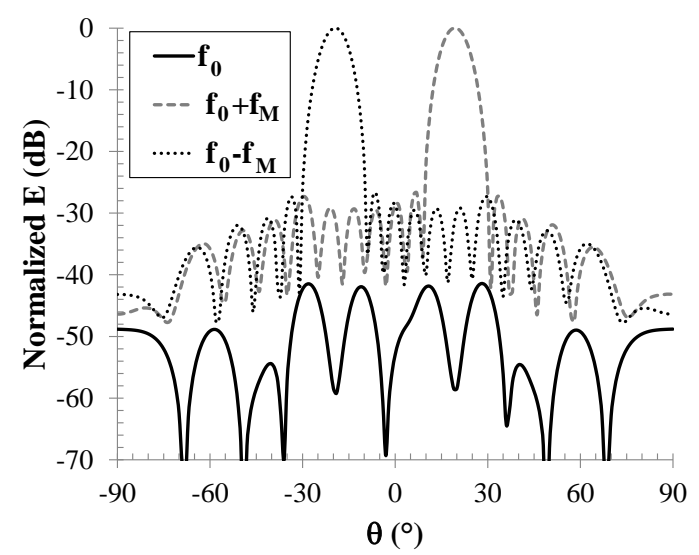

Fig. 17. Fundamental and first sideband harmonics patterns of the array of Fig. 10(a), under excitation conditions of Fig. 16.

\section{MEASURED Results}

As an experimental proof of the TMA technique, we have prototyped a two-element array to be driven by the control sequence used in the first step of operation to generate the $\Sigma$ and $\Delta$ patterns for localizing the tags to be energized. Since for this purpose the simulated performance of the arrays with element spacing equal to $\lambda / 2$ and $\lambda / 8$ are similar, we decide for the standard larger array and we co-design the array with its feeding network. The final layout of the realized prototype is shown in Fig. 18. The adopted SMD components for each array element feeding network of the kind of Fig. 1 are: two Schottky diodes (Skyworks SMS7630-079), two $10 \mathrm{pF}$ dc block capacitors (Murata GRM1885), four RF chokes $\left(\mathrm{L}_{1}=\mathrm{L}_{2}\right.$ $=15 \mathrm{nH})$ (Johanson Technology L402DC), and two $1 \mathrm{k} \Omega$ bias resistors. A microprocessor TI MSP430 is used to drive the diodes based on the control patterns of Fig. 5, with $f_{M}=25$ $\mathrm{kHz}$. Fig 19 shows the real waveforms measured at the microprocessor ports, for the cases $\mathrm{d}=0 \%$ and $\mathrm{d}=32 \%$. In order to properly bias the Schottky diodes, positive and 


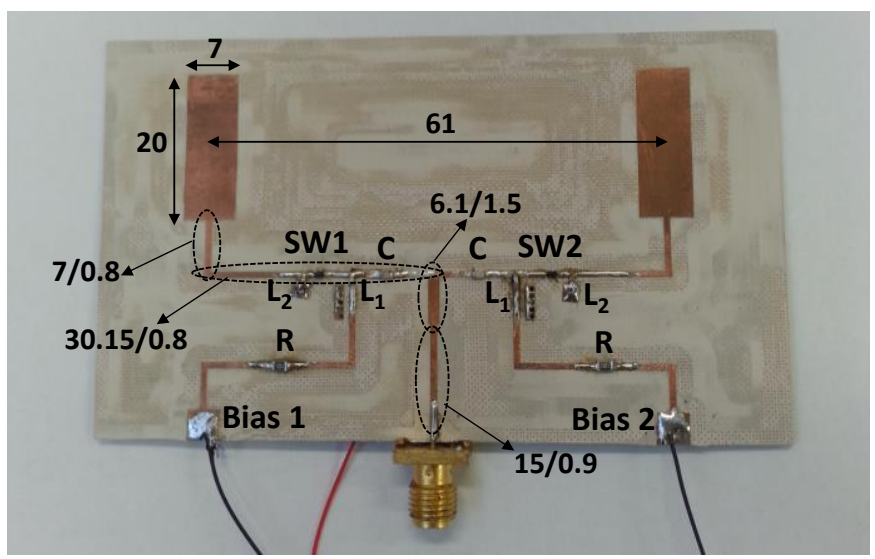

Fig. 18. $2.45 \mathrm{GHz}$ prototype of a two-element TMA, with dimensions in $\mathrm{mm}$ (lines dimensions: length/width).

negative waveforms levels are $-3.5 \mathrm{~V}$ and $3.5 \mathrm{~V}$, respectively, thus ensuring the correct control at the antenna ports. The waveforms show slight oscillations around the $\pm 3.5 \mathrm{~V}$ values, while the zoomed inset demonstrates the rapid transition between the two states $\left(\mathrm{t}_{\text {rise }}=\mathrm{t}_{\text {fall }} \approx 60 \mathrm{~ns}\right)$. A $3.5-\mathrm{mA}$ current flows through the diodes in on-state.

Since the actual waveform driving the diodes is the crucial design parameter in TMA synthesis, the measured control waveforms are first used inside the system simulation to verify the actual modelled system performance: the corresponding simulated $\Sigma$ and $\Delta$ radiation patterns result to be almost identical to those of Fig. 9(a), obtained with ideal rectangular pulses, apart from a difference of a few $\mathrm{dB}$ in correspondence of the $\Delta$ negative peaks, thus confirming the good quality of the measured switch bias patterns.

Fig. 20 shows the photo of the measurement set-up prepared in an office scenario to test the system in real operating conditions.

The first test of the prototype consists of spectra
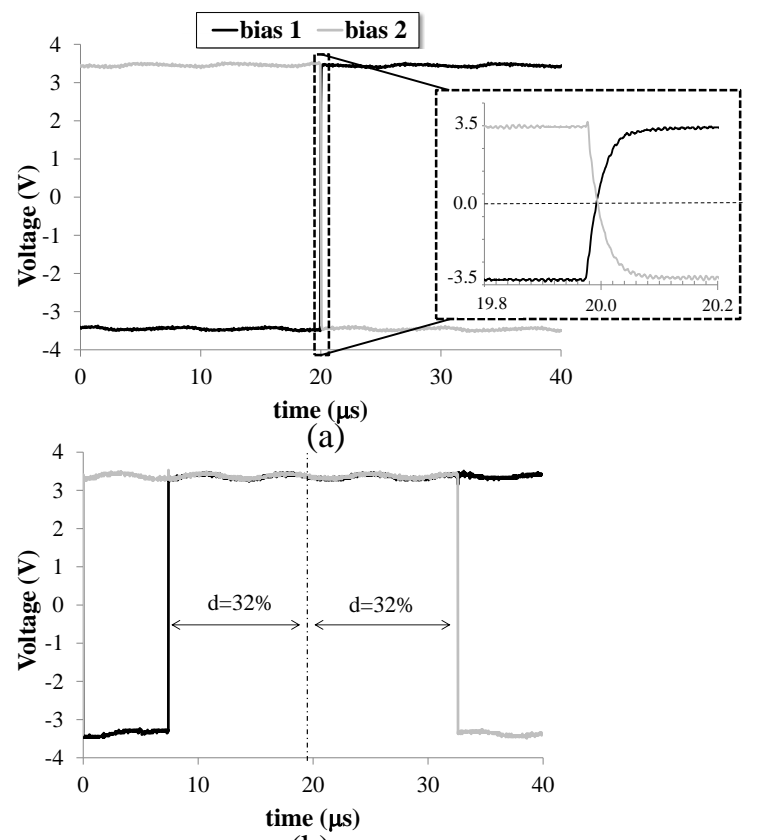

(b)

Fig. 19. Measured pulse waveforms at the TI MSP430 output ports, (a) with the tuning parameter $\mathrm{d}=0 \%$, and (b) with $\mathrm{d}=32 \%$.

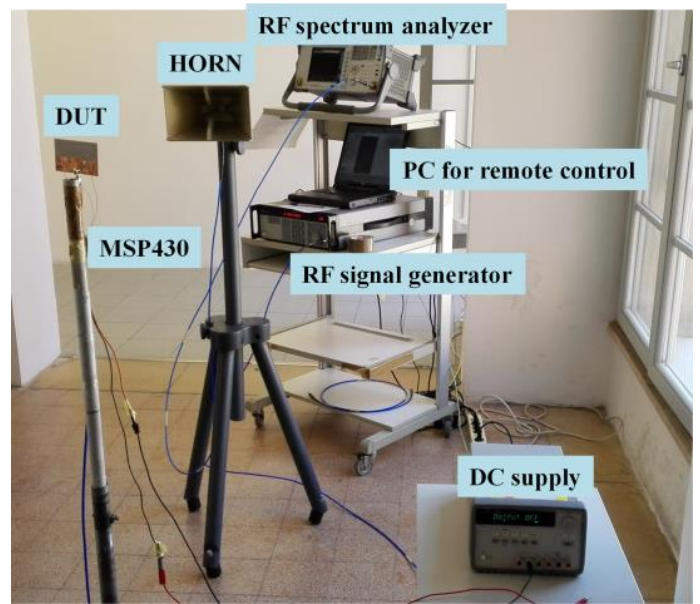

Fig. 20. Photo of the measurement set-up.

measurements to verify the complex radiation mechanism of the system under exam: the measured spectra obtained by switching the diodes with the sequence of Fig. 19(a, b) are reported in Fig. 21 for the broadside direction $\left(\theta=0^{\circ}\right)$, and for $\theta=30^{\circ}$ and $45^{\circ}$. The sideband radiation phenomenon at $2.499975 \mathrm{GHz}$ and $2.450025 \mathrm{GHz}$ is clearly visible in the figure: in the perfect complementary excitation case $(\mathrm{d}=0 \%)$ the $\Delta$ field strength, at the sideband harmonics, increases with increasing $\theta$ value, whereas the $\Sigma$ field at the fundamental smoothly changes, as predicted by simulation. In the shifted control sequence case $(\mathrm{d}=32 \%)$, the $\Delta$ field has a reverse dependence on $\theta$, and its minimum is around $\theta=45^{\circ}$, in this case. From this preliminary results it is already notable that the $\Delta$ minimum in the $\mathrm{d}=32 \%$ case is not very deep, since the sideband harmonic spectral lines are not much lower than the fundamental one (see Fig. 21(c)).

The resulting measured radiation patterns are shown in Fig. 22 for $\mathrm{d}=0 \%, 32 \%$, confirming that the TMA operation is reached. The $\Sigma$ and $\Delta$ patterns have the predicted shape: in

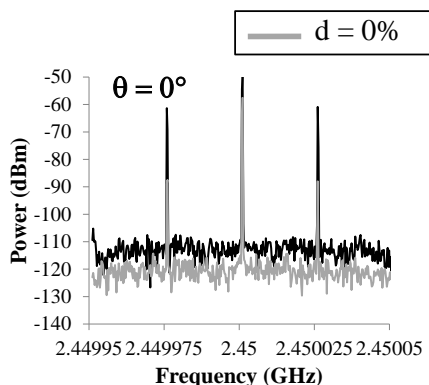

(a)

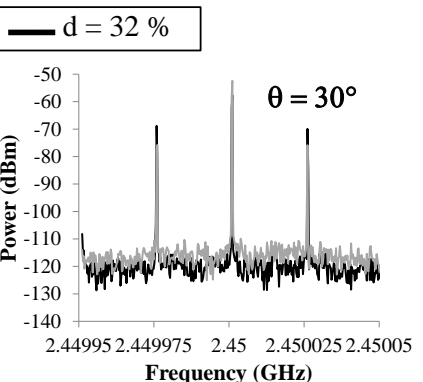

Frequency $(\mathbf{G H z})$

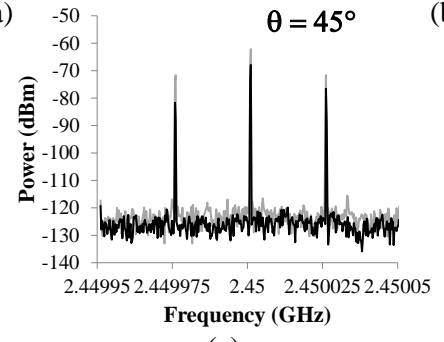

(c)

Fig. 21. Measured fundamental and first sideband harmonics spectral lines, radiated in three different directions: (a) $\theta=0^{\circ}$, (b) $\theta=30^{\circ}$, (c) $\theta=45^{\circ}$, in the case of the tuning parameter $\mathrm{d}=0 \%, 32 \%$. 
particular a very deep peak $(-40 \mathrm{~dB})$ of the $\Delta$ pattern with $\mathrm{d}=$ $0 \%$ is reached, and good steering capabilities pertain to the $\mathrm{d}>$ $0 \%$ case (the $\Delta$ peaks are $-25 \mathrm{~dB}$, only, for $\mathrm{d}=32 \%$ ). The $\Sigma$ pattern is not reported for all the d-values because it is always the same, due to its un-steerable nature.

Few discrepancies with simulated results are observed and are discussed below. A slight asymmetry of the measured $\Delta$ patterns can be observed: in particular, when $\mathrm{d}=0 \%$, the negative peak is at $\theta \approx-10^{\circ}$ (expected $0^{\circ}$ ) for both the harmonics. This is probably due to the in-house realization of the prototype, which could be responsible for asymmetries in the feeding network and/or in the antenna layouts, causing the phase unbalance at the antenna ports. This is also confirmed by the patterns in the $\mathrm{d}=32 \%$ case: the peaks are at $\theta \approx-50^{\circ}$ (expected $-45^{\circ}$ ) and $\theta \approx 40^{\circ}$ (expected $45^{\circ}$ ), for the higher and lower sideband, respectively.

Furthermore a lower amplitude of the measured $\Delta$-field with respect to the corresponding modelled one is observed. This is probably due to the uncertainties of the available diode package model used in the nonlinear simulation of the entire system. Indeed we experimentally observed that in real operation an alternative path for the RF signal is created by the diode capacitance parasitic, which allows a small amount of the RF signal to reach the antenna ports, thus perturbing the delicate equilibrium which the sideband radiation in based on.

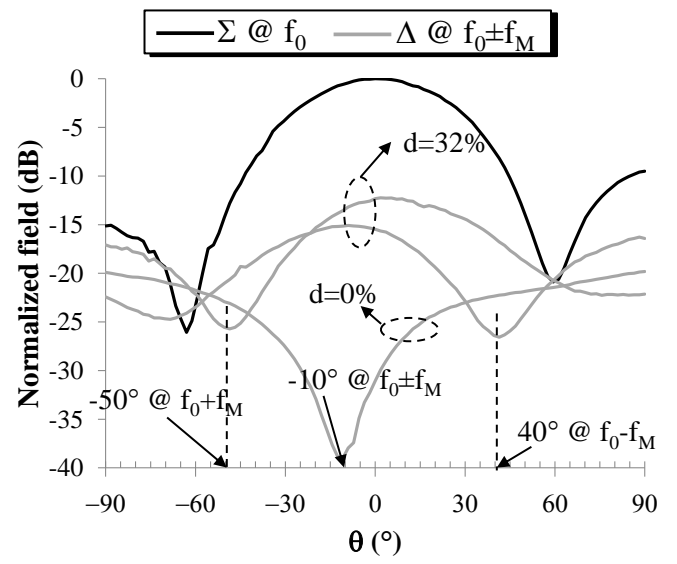

Fig. 22. Measured $\Sigma$ (at fundamental) and $\Delta$ radiation patterns for $\mathrm{d}=0 \%$, $32 \%$ (at first sideband harmonics).

\section{CONCLUSION}

In this paper, we exploit the unrivaled level of configurability of TMAs to perform a new dynamic two-step intentional WPT operation. By simultaneously controlling in real-time the bias waveforms of nonlinear switches feeding the array elements, we show that the system allows to first precisely localize and then energize randomly placed sensors, without wasting of power. The actual nonlinear switch dynamics and the linear EM couplings between different portions of the WPT system are rigorously predicted by the presented co-design procedure, demonstrating its necessary role for the accurate prediction of TMAs implementation. The effectiveness of the adopted design method is demonstrated by the measured radiation patterns of a realized two-monopole array: the obtained behavior demonstrates the feasibility of the TMAs localization function, even in a realistic office scenario.
These first promising results, obtained by very simple architecture implementation, indicate TMAs as very interesting candidates for agile and reconfigurable WPT systems, to be exploited in many civil and industrial energywise assisted-ambient. Future work will be dedicated to the implementation and experimental tests of TMA-based WPT systems, for different requirements in terms of power levels.

\section{REFERENCES}

[1] M. Pinuela, P.D. Mitcheson, S. Lucyszyn, "Ambient RF Energy Harvesting in Urban and Semi-Urban Environments," IEEE Trans. Microw. Theory Techn., vol.61, no.7, pp.2715-2726, July 2013.

[2] D. Masotti, A. Costanzo, M. Del Prete, V. Rizzoli, "A Genetic-Based Design of a Tetra-Band High-Efficiency RF Energy Harvesting System", IET Microw. Antennas Propag., vol.7, no.15, pp.1254-1263, 2013.

[3] J.A. Hagerty, F.B. Helmbrecht, W.H. McCalpin, R. Zane, Z.B. Popovic, "Recycling ambient microwave energy with broad-band rectenna arrays", IEEE Trans. Microw. Theory Techn.., vol.52, no.3, 2004, pp.1014-1024.

[4] A. Massa, G. Oliveri, F. Viani, P. Rocca, "Array Designs for LongDistance Wireless Power Transmission: State-of-the-Art and Innovative Solutions," Proceedings of the IEEE, vol.101, no.6, pp.1464-1481, June 2013.

[5] E. Hossain, M. Rasti, H. Tabassum, A. Abdelnasser, "Evolution toward 5G multi-tier cellular wireless networks: An interference management perspective," IEEE Wireless Comm. vol.21, no.3, pp.118-127, June 2014.

[6] L. Roselli, N. Borges Carvalho, F. Alimenti, P. Mezzanotte, G. Orecchini, M. Virili, C. Mariotti, R. Goncalves, P. Pinho, "Smart Surfaces: Large Area Electronics Systems for Internet of Things Enabled by Energy Harvesting," Proceedings of the IEEE, vol.102, no.11, pp.1723-1746, Nov. 2014.

[7] M.M. Tentzeris, Sangkil Kim, R.Vyas, A. Traille, P. Pons, H. Aubert, A. Georgiadis, A. Collado, "Inkjet-printed "Zero-Power" wireless sensor and power management nodes for IoT and "Smart Skin" applications," 20th Int. Conf. MIKON, pp.1-7, June 2014.

[8] J.A. Hagerty, F.B. Helmbrecht, W.H. McCalpin, R. Zane, Z.B. Popovic, "Recycling ambient microwave energy with broad-band rectenna arrays," IEEE Trans. Microw. Theory Techn., vol.52, no.3, pp.10141024, March 2004

[9] A. Boaventura, D. Belo, R. Fernandes, A. Collado, A. Georgiadis, N. Borges Carvalho, "Boosting the Efficiency: Unconventional Waveform Design for Efficient Wireless Power Transfer," IEEE Microwave Magazine, vol.16, no.3, pp.87-96, April 2015.

[10] S. Yoshida, N. Hasegawa, S. Kawasaki, "Experimental Demonstration of Microwave Power Transmission and Wireless Communication Within a Prototype Reusable Spacecraft," IEEE Microw. Wirel. Comp. Lett., to be published.

[11] A. Costanzo, M. Dionigi, D. Masotti, M. Mongiardo, G. Monti, L. Tarricone, R. Sorrentino, "Electromagnetic Energy Harvesting and Wireless Power Transmission: A Unified Approach", Proceedings of the IEEE , vol.102, no.11, pp.1692-1711, Nov. 2014.

[12] D. Masotti, R. Marchukov, V. Rizzoli, A. Costanzo, "Far-field power transmission by exploiting time-modulation in linear arrays," Proc. 2015 IEEE WPTC, Boulder (CO), pp.1-4, 2015.

[13] H. E. Shanks, R. W. Bickmore, "Four dimensional electromagnetic radiators," Canadian J. of Physics, vol.37, no.3, pp. 263-275, 1959.

[14] W. H. Kummer, A. T. Villeneuve, T. S. Fong, F. G. Terrio, "Ultra-low sidelobes from time-modulated arrays," IEEE Trans. Antennas Propag., vol.AP-11, no.6, pp.633-639, Nov. 1963.

[15] Y. Chen, S. Yang, G. Li, Z. Nie, "Adaptive nulling with time-modulated antenna arrays using a hybrid differential evolution strategy," Electromagnetics, vol.30, no.7, pp.574-588, Sept. 2010.

[16] G. Li, S. Yang, Y. Chen, Z. Nie, "An adaptive beamforming in time modulated antenna arrays", Proc. ISAPE 2008, pp.166-169, Nov. 2008.

[17] L. Poli, P. Rocca, G. Oliveri, A. Massa, "Harmonic beamforming in time-modulated linear arrays" IEEE Trans. Antennas Propag., vol.59, no.7, pp.2538-2545, July 2011.

[18] Y. Tong, A. Tennant, "Simultaneous control of sidelobe level and harmonic beam steering in time-modulated linear arrays," Electronics Lett., vol.46, no.3, Feb. 2010. 
[19] G. Li, S. Yang, Y. Chen, Z. Nie, "A novel electronic beam steering technique time modulated antenna arrays," Progr. Electromag. Res., vol.97, pp.391-405, 2009.

[20] A. Tennant, B. Chambers, "A Two-Element Time-Modulated Array With Direction-Finding Properties", IEEE Antennas Wirel. Propag. Lett., vol.6, pp. 64-65, 2007.

[21] G. Li, S. Yang, Z. Nie, "Direction of arrival estimation in time modulated antenna arrays with unidirectional phase center motion," IEEE Trans. Antennas Propag., vol.58, no.4, pp.1105-1111, Apr. 2010.

[22] H. Chong, L. Xianling, L. Zhaojin, G. Junping, J. Ronghong, "Direction finding by time-modulated array with harmonic characteristic analysis", IEEE Antennas Wirel. Propag. Lett., vol.14, pp.642-645, 2015.

[23] P. Rocca, Q. Zhu, E.T. Bekele, S. Yang, A. Massa, "4-D Arrays as Enabling Technology for Cognitive Radio Systems," IEEE Trans. Antennas Propag., vol.62, no.3, pp.1102-1116, March 2014.

[24] T. Takahashi, T. Mizuno, M. Sawa, T. Sasaki, T. Takahashi, N. Shinohara, "Development of phased array for high accurate microwave power transmission," 2011 IEEE MTT-S IMWS, pp.157-160, May 2011.

[25] R.T. Iwami, A. Zamora, T.F. Chun, M.K. Watanabe, W.A. Shiroma, "A retrodirective null-scanning array", 2010 IEEE MTT-S IMS Digest, pp.81-84, May 2010.

[26] S. Yang, Y. B. Gan, A. Qing, P. K. Tan, "Design of a uniform amplitude time-modulated linear array with optimized time sequences," IEEE Trans. Antennas Propag., vol.53, no.7, pp.2337-2339, July, 2005.

[27] H. Chong, Y. Hangchen, L. Xianling, G. Junping, J. Ronghong, "Sideband Radiation Level Suppression in Time-Modulated Array by Nonuniform Period Modulation", IEEE Antennas and Wireless Propagation Lett., vol.14, pp.606-609, 2015

[28] A. Tennant, "Experimental Two-Element Time-Modulated Direction Finding Array", IEEE Trans. Antennas Propag., vol.58, no.3, pp.986988, March 2010.

[29] M. Del Prete, D. Masotti, N. Arbizzani, A. Costanzo, "Remotely Identify and Detect by a Compact Reader With Mono-Pulse Scanning Capabilities," IEEE Trans. Microw. Theory Techn., vol.61, no.1, pp.641650, Jan. 2013.

[30] D. Masotti, P. Francia, A. Costanzo, V. Rizzoli, "Rigorous Electromagnetic/Circuit-Level Analysis of Time-Modulated Linear

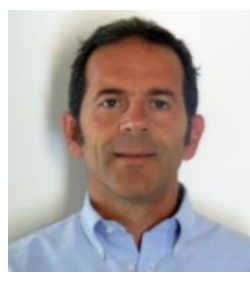

Diego Masotti ( $\left.\mathrm{M}^{\prime} 00\right)$ received the Ph.D. degree in electric engineering from the University of Bologna, Italy, in 1997. In 1998 he joined the University of Bologna as a Research Associate of electromagnetic fields. His research interests are in the areas of nonlinear microwave circuit simulation and design, with emphasis on nonlinear/electromagnetic co-design of integrated radiating subsystems/systems for wireless power transfer and energy harvesting applications. Dr. Masotti serves in the Editorial Board of the International Journal of Antennas and Propagation, and is a member of the Paper Review Board of the IEEE Transactions on Microwave Theory and Techniques since 2004.

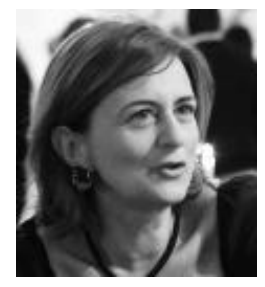

Alessandra Costanzo (M'99-SM'13) is Associate professor of electromagnetic fields at the University of Bologna, Italy since 2001. Her main research interests include multi-domain design (based on nonlinear/electromagnetic co-simulation) of entire wireless links, such as RF-ID, MIMO and UWB, including rigorous modeling of radiating elements and realistic channel models. Recently she has developed innovative wireless power systems for both far- and near-field solutions. She co-authored more than 150 scientific publications on peer reviewed international journals and conferences and three chapter books; she holds two European and one US patents. She is associate editor of the Cambridge Wireless Power transfer journal and International Journal of Microwave and Wireless Technologies. In 2013 she co-funded the EU COST action WiPE "Wireless power transfer for sustainable electronics" where she chairs WG1: "far-field wireless power transfer". Prof. Costanzo is the chair of the IEEE MTT-26 Technical Committee on Wireless Energy Transfer and Conversion, and MTT-S representative of the IEEE Council on RFID.
Arrays," IEEE Trans. Antennas Propag., vol.61, no.11, pp.5465-5474, Nov. 2013

[31] L. Poli, P. Rocca, L. Manica, and A. Massa, "Pattern synthesis in timemodulated linear arrays through pulse shifting," IET Microw., Antennas Propag., vol.4, no.9, pp.1157-1164, Sept. 2010.

[32] Q. Zhu, S. Yang, L. Zheng, Z. Nie," Design of a Low Sidelobe Time Modulated Linear Array With Uniform Amplitude and Sub-Sectional Optimized Time Steps," IEEE Trans. Antennas Propag., vol.60, no.9, pp.4436-4439, Sept. 2012.

[33] Y. Jing, L. Wen-Tao, S. Xiao-Wei, X. Li, Y. Jian-Feng, "A Hybrid ABC-DE Algorithm and Its Application for Time-Modulated Arrays Pattern Synthesis," IEEE Trans. Antennas Propag, vol.61, no.11, pp.5485-5495, Nov. 2013.

[34] J. Euziere, R. Guinvarc'h, B. Uguen, R. Gillard, "Optimization of Sparse Time-Modulated Array by Genetic Algorithm for Radar Applications," IEEE Antennas Wirel. Propag. Lett., vol.13, pp.161-164, 2014.

[35] E.T. Bekele, L. Poli, P. Rocca, M. D'Urso, A. Massa, "Pulse-Shaping Strategy for Time Modulated Arrays-Analysis and Design," IEEE Trans. Antennas Propag., vol.61, no.7, pp.3525-3537, July 2013.

[36] V. Rizzoli, D. Masotti, F. Mastri, E. Montanari, "System-Oriented Harmonic-Balance Algorithms for Circuit-Level Simulation", IEEE IEEE Trans. Comput.-Aided Design. Integr. Circuits Syst., vol.30, no.2, pp.256-269, Feb. 2011.

[37] V. Rizzoli, A. Costanzo, D. Masotti, P. Spadoni, A. Neri,"Prediction of the End-to-End Performance of a Microwave/RF Link by Means of Nonlinear/Electromagnetic Co-Simulation," IEEE Trans. Microw. Theory Techn., vol.54, no.12, pp. 4149-4160, Dec. 2006.

[38] P. Rocca, L. Poli, A. Massa, "Instantaneous directivity optimisation in time-modulated array receivers", IET Microw., Antennas Propag., vol.6, no.14, pp.1590-1597, Nov. 2012.

[39] S. Yang, Y. B. Gan, A. Qing, "Sideband suppression in time-modulated linear arrays by the differential evolution algorithm", IEEE Antennas Wirel. Propag. Lett., vol.1, pp.173-175, 2002.

[40] A-Min Yao, Wen Wu, Da-Gang Fang, "Single-Sideband TimeModulated Phased Array," IEEE Trans. Antennas Propag., vol.63, no.5, pp.1957-1968, May 2015.

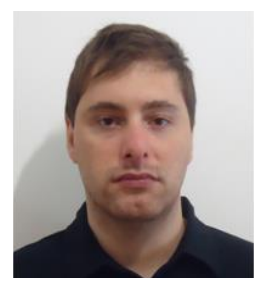

Massimo Del Prete received the B.S. and M.S. degree in telecommunication engineering at the University of Bologna, Italy, in 2007 and 2011, respectively. In 2014 he joined the Department of Electrical, Electronic and information Engineering, University of Bologna as a $\mathrm{PhD}$ student. His research interests include wearable and multi-band antennas, CAD of microwave integrated circuits, with special emphasis on low-power rectenna, power management for autonomous sensors, and wireless power transmission.

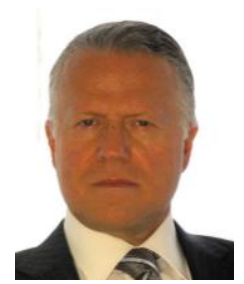

Vittorio Rizzoli (M'79-SM'90-F'94) joined the University of Bologna as a Full Professor of Electromagnetic Fields in 1980. His main research interests are in the areas of nonlinear microwave circuit simulation and design with emphasis on electromagnetic analysis and design of microwave integrated circuits, and nonlinear/electromagnetic cosimulation and co-design of communications links. He is author or co-author of over 210 refereed technical papers on these and related topics.

Dr. Rizzoli is a member of the Editorial Board of the IEEE Transactions on Microwave Theory and Techniques, of IEEE Microwave and Wireless COMPONENTS LETTERS, and of John Wiley's International Journal of RF and Microwave Computer Aided Engineering. In 1990/92 he served as the Distinguished Microwave Lecturer of IEEE MTT-S for Region 8, lecturing in Europe, U.S.A. and Middle East on "Simulation and Design of Nonlinear Microwave Circuits. Since 1993 he has been a member of the Technical Committee IEEE MTT-1 on Computer-Aided Design. From 1999 to 2009 he was a member of the Technical Program Committee of the IEEE MTT-S International Microwave Symposium. In 2005/2006 he served as an Associate Editor for the IEEE Transactions on Microwave Theory and Techniques 